\title{
An Exploration on English Learning and Teaching Based on the Theory of Figure and Ground
}

\author{
Yuanzhen $\mathrm{Wu}$ \\ Gongqing College Nanchang University \\ Gongqingcheng, China 332020
}

\author{
Shijun Liu \\ Gongqing College Nanchang University \\ Gongqingcheng, China 332020
}

\begin{abstract}
Originated from psychology, the theory of Figure and Ground was integrated by gestalt psychologists to the framework of perceptual organization and currently it has been put into use in the analysis of linguistic structure by cognitive linguists, especially in the construal of prepositions and complex sentences indicating temporal events. In addition, this theory could serve to explore its guidance in English teaching and the switch of teachers' role in the process of teaching.
\end{abstract}

Keywords-figure-ground; prepositions; temporal events; English teaching

\section{INTRODUCTION}

Cognitive linguistics mainly employs three methods to characterize language (Wenxu 2004): experiential view, attentional view and prominence view. According to the experiential view, people's description of things is not only limited to objective description, but also provides richer and more natural interpretation of their meaning, including metaphor. According to the attention view, what we express in language actually reflects only those parts of the event that attract our attention. According to the highlighting view, the choice and arrangement of information in the language structure are determined by the highlighting degree of information. And the theory of Figure and Ground is based on the theory of highlighting, which can not only be applied to the psychological research, but also can be used in cognitive linguistics as a reference to analyze language structure, and it plays a guiding role in English teaching as well.

\section{THEORETICAL BASIS}

The theory of Figure and Ground was first put forward nearly a century ago by psychologist Denmark Rubin through the famous "illusion" face/vase experiment (Ungerer \& Schmid, 2001:157). He argued that it was impossible to identify face and vase at the same time, which is called "figure- ground separation principle" (figure - ground segregation). This theory was employed by Gestalt psychologists as a reference to the study of perceptual field. They were very interested in how visual and auditory input had impact on this issue based on the highlighting principle, which focuses on the whole, and they thought that perceptual field had always been divided into two parts, namely figure and ground. The perceptual field in figure is a highlight in cognitive concepts or perception, the focus of cognition which attracts people's attention while the ground poses the contrast with figure that contains vague concepts and serves as a foil to figure, and it acts as a cognitive reference to figure. When people observe an object, they see a figure in an undifferentiated ground. In cognitive linguistics, Langacker employed trajector and landmark instead of the terms of figure and ground. If an object is in a position relative to another object or moves towards the latter, the former is called the trajector and the latter is the landmark.

M.Wetheimer, German gestalt psychologist, proposed the law of Pragnanz, which is one of the most basic law of consciousness, also called law of containing. (Ungerer \& Schmid, 2001:34). According to it, the figure is characterized by small or moving object of gestalt. In other words, people have tendency to present what they perceive in the best possible way, namely gestalt. If a person's field of perception is disturbed, he immediately forms a new field of perception so that there is still a good form of what is perceived. It should be noted that this perfect form does not refer to the best one, but emphasizes its integrity. This process is the process of perceptual restructuring. Its operation contains five perceptual laws:

- 1aw of proximity, indicating that people's consciousness is combined based on the degree of closeness and adjacence of each part to another. The closer the parts are, the more likely they are combined together;

- 1aw of similarity, indicating that when people perceive items of similar stimulus elements, they tend to combine them as long as they are not disturbed by adjacent factors. In other words, similar parts form several combinations in perception;

- 1aw of closure indicating that closed patterning in the field of vision are easy to form figure;

- 1aw of continuity, that is, in the process of perception, people tend to maintain the original form of the object of perception, where the line continues to be a straight line and the curve continues to be a curve;

- 1aw of the membership character, indicating that according to gestalt psychologists, the individual parts of a whole does not have fixed characteristics 
and the characteristics of individual parts are shown from the relationship between these and other parts.

\section{FIGURE AND GRound PRESENTEd In LANGUAGE}

Traditional linguistics focuses mainly on the level of grammatical rules of language, while cognitive linguistics believes that the experience structure and cognitive mode of human also play a significant role in language interpretation. And the figure - ground separation principle can be used in the study of the language structure due to the fact that language expression is based on people's perception and arrangement of things or events, and figure and ground are organized to express the language. The perception of figure and ground is the direct result of human experience, because people will always use an object or concept in our daily life as the cognitive reference point to explain or illustrate another object or concept while the ground here is the cognitive reference point of figure.

The first scholar who applied figure and ground theory in language study is Talmy, who thought the figure - ground separation principle is a basic cognitive principle of organization, and cited respectively the definition characteristics and association characteristics of figure and ground in language (Talmy2000:315-316) :

TABLE I. FIGURE AND GROUND IN LANGUAGE

\begin{tabular}{|l|l|}
\hline Figure & Ground \\
\hline unknown space & known space \\
\hline Small area or volume & Large area or volume \\
\hline More mobile & Fixed position \\
\hline Simple structure & More complicated structure \\
\hline High visibility & Less prominence \\
\hline $\begin{array}{l}\text { Closer in consciousness; High } \\
\text { correlation }\end{array}$ & $\begin{array}{l}\text { Further in a situation or memory; } \\
\text { Low correlation }\end{array}$ \\
\hline
\end{tabular}

These characteristics are limited to the field of spatial relations, which have been modified and supplemented by Kuang Fangtao and Wen Xu (2003). The definition features of figure and ground lie in the fact that figure could not determine known spatial or temporal characteristics while the ground has known spatial or temporal characteristics, which can be used as reference points to describe and determine the unknown features of the figure. At the same time, five descriptive dimensions have been added to the association features, such as time span, accessibility, dependence, familiarity and predictability.

\section{A. The Interpretation of Prepositions}

The meaning of the preposition of orientation can be understood as a figure-ground relationship. The commonly used preposition are on/above/over.; under/below; in front of; behind; by/fit/near. In a simple sentence, more often than not the noun in a preposition phrase is the ground and the other noun is the figure. For example:

- (1) A cup is on the table.

- (2) There is a chair in the house.
In both examples, the cup and chair are figures, and the table and house are used as reference points for ground. Since cups and chairs are smaller, simpler and more prominent than tables and houses, they are seen as figures. Compared with cup and chair, table and house take up more space, with more complex structure and less degree of prominence, so they can be regarded as ground. All of these conform to the law of Prägnanz and the definition and association characteristics of figure and ground. That is to say, whatever angle an observer takes, the glass is still on the table, and a chair is in the room and there is no case that the table and the house serve as a figure, while cup and chair is ground. For instance:

- (3) A table is under the cup.

- (4) There is a house outside the chair.

These two sentences are against the 1aw of Prägnanz and also the laws of cognition, which sound strange. This is what we call the asymmetrical relationship between figure and ground, which in turn is not true. There are, of course, not to say that these two sentences are completely unacceptable. It might make sense in a specific context, just like in the third example. Is it possible that there is such a situation: some sort of tables are small enough, and some cups are big enough so that a glass cup is greater than a table, which makes us naturally regard the cup as the ground, the glass table as figure? Although this situation is almost impossible, we cannot completely deny its existence. We can see the first two examples here as a typical member of the general category of simple sentence following the law of Pragnanz and the asymmetrical examples 3 and 4 as a marginal member of the category.

For another example, the following two sentences of a.b, in which the figure and ground are symmetric and they are equal in the true value condition while it does not mean that they are semantically equivalent:

- (5) a. Jim is near Lucy. b. Lucy is near Jim.

Obviously, we can find that the two sentences listed above are different from previous sentences, because in the first sentence Jim is figure and Lucy is ground while in the second sentence their positions are reversed. On the other hand, this pair of sentences is symmetrical and both can be accepted. This is because in the absence of specific context, the gap between the figure and the ground in all aspects is unknown, or as a rule, people mentioned in the sentences are similar with no big difference, which is different from the case above, so the law of Prägnanz could not account for this phenomenon strongly enough. In this case, the figure and the ground are interchangeable, that is, with symmetry. However, it does not mean that these two sentences are completely equal. They are semantically different, which can be considered from the perspective of defining features. In the first sentence Lucy is the reference point of Jim, namely determining the position of the Jim by Lucy. From this we can assume that such a situation: in a classroom, student A tries to find Jim's seat, but student B only knows Lucy's position A, and then B said Jim is near Lucy, so it is easy for A to find Jim, without specifically saying the line and row. 
Similarly, (5b) is the opposite, that is, determining Lucy's position from Jim, the reference point. In short, the ground is known and the figure is unknown, and the unknown can be construed from the known.

However, we can note that not all figures and grounds are specific, such as:

- (6) The future is in front of us.

- (7) Harry is in love. (Lakoff 1980:59)

According to defining characteristics, in the sixth example, the future is viewed from the perspective of us, and thus us is the ground, while the unknown future is figure. More often than not, people only know the past or the moment, without predicting what will happen tomorrow. The example is different from the above examples, for people could neither see nor touch abstract concepts like the figure future with unboundedness and unpredictability, but in this sentence the future is externalized and metaphorized as an object standing in front of us. The ground love in the seventh sentence is abstract, and it belongs to the category of feelings without indicating time and space, and therefore it is inconsistent with the definition characteristics of ground. Instead it possesses metaphorical senses in prepositional phrase, namely comparing love to a container. In other words, Harry is in the container of love, however the meaning of the preposition does not change, and in love conveys an emotional state.

\section{B. Analysis of Complex Sentences Representing Time Events}

According to the degree of highlighting, Langacker (1987:120) made a discussion on figure and ground: the figure in a ground is a secondary structure, more prominent than the other parts of ground, and as a central entity it has a special highlight, with scene organized around it, providing an environment for it. In addition, a relatively dense area in sharp contrast to the environment is more likely to be chosen as a figure. This contains the concept of inner inclusion, that is, the ground containing the figure. After that, Talmy clearly proposed the inclusion principle and contingency principle (Liu Guo-hui 2006). The inclusion principle refers to that the big events with time inclusion are usually taken as the ground and placed in clauses, and the inclusive events in the main clause are regarded as the figure; The dependency principle refers to the event that determines another event in the clause serves as the ground, and the event that is attached to the clause event in the main clause as the figure. These two principles have strong explanatory power for complex sentences expressing time events, for instance:

- (8) a. He dreamed while He slept. b. *He slept while He dreamt.

This typical example was put forward by Talmy (2000:325). In general, it is known that compared with dream, sleep lasts longer, and in other words, dream is the phenomenon of what happens during sleep, so it is included in sleep in terms of time length. It follows that dream, the figure, appears in the clause, while the bed, the ground appears in the main clause. This is in line with the inclusion principle, from which we know that dreaming is included in the big event of sleep; on the other hand, from the principle of interdependence, usually in the true sense dreaming does not exist alone; in other words, sleep decides the existence of a dream, and a dream highlights in this event of sleep, naturally serving as figure. This is why $(8 a)$ is acceptable and (8b) is counterintuitive, because the event of dreaming does not outweigh and determine the existence of sleep. In fact, this is also the asymmetry mentioned above, and the figure and ground do not switch. However, we often can find the switch of figure and ground in some complex sentences indicating time events more flexible:

- (9) a. He walked while he listened to the music. b. He listened to the music while He walked.

Both examples are acceptable, and the figures and grounds of the first and second sentence are switched and symmetrical. In general, listening to music is more prominent, therefore more appropriate as a figure, and walking is a larger event, serving as a ground. However, this is not necessarily true, because walking and listening to music can be simultaneous, excluding the possibility of one event containing another. In other words, other things can be done during walking and listening to music can also be associated with other events. It should be noted that the connotation of these two sentences are not exactly the same: in (9a) the walking event is highlighted and in (9b) the music event is emphasized.

\section{CONCLUSION}

\section{A. Implication in English Teaching}

In simple sentences, the subject and object or complement can be regarded respectively as the indication of the figure and ground, and the predicate reflects the relationship between them. According to the definition and associative features, the subject is unknown and people are not familiar with it while in contrast, the object is known. In the practice of reading comprehension, it is often required to summarize the main idea, and as a rule, if a word or phrase in a discourse always appears in the position of subject, it acts as the figure, so it can be concluded that this article is mainly about something the word or phrase refers to. However, a large number of sentences do not fall into this category, and we can often meet them when the ground is in the front and the figure is in the back, such as inverted sentences and passive sentences. In inverted sentences, usually the ground is in the position of the subject; for example, the focus of the sentence on the desk is a book is the book, which moves back, whereas the ground moves forward. It is to highlight the ground, the unknown table, and the subject of a book is known at the end of the sentence. In passive sentences, e.g. the clothes were washed by Mary, the clothes becomes the subject instead of the object in the original sentence, and acts as the figure. Correspondingly, predicate verb is changed, and so these two sentences are asymmetric. In short, the two typical sentence patterns discussed above have something in common, that is, the order of the known to the unknown, the old information to 
new information. Therefore, in writing, we need not be constrained by the linear structure of figure to ground or subject to object; instead in the layout of an essay we can flexibly place focus, employing different sentence patterns, in order to make structure compact yet dynamic.

\section{B. Change in the Role of Teachers}

Zhong Meisun (2014) pointed out that China's popularization of higher education has a significant impact on higher foreign language education results in the lack of features of cultivating talented college students and the problem of unguaranteed teaching quality. One of the main reasons for this is that traditional teachers act as the leading role, and the lack of interaction between teachers and students in class hinders the further improvement of English teaching at present. This is because students are always in the passive state of accepting knowledge, and lots of input and little output restrains timely feedback by students in the classroom. Despite the rise of new teaching models such as flipped classroom in recent years, many ordinary colleges and universities in less developed areas still adopt traditional teaching models.

Combined with the theory of figure and ground, we can consider the relationship between teachers and students in class as the figure and the ground. In such a relationship the teacher is the focus, free to "move" while students in class are the ground. Therefore, it is necessary to change the teaching mode and switch the position of figure and ground, namely student-figure, teacher-ground. It emphasizes the students' participation and the teacher's leading role; in other words, students become the focus in class, and are given more opportunities to convert input into output. Harmer (2000) pointed out that two or three students were asked to form a group to discuss a topic. During the discussion, teachers did not participate but wrote down students' mistakes. When the discussion goes on for a certain period of time and the students have difficulties to continue, the teacher can intervene, correct the mistakes and guide the discussion. It can be seen that in the previous stage, the teacher, as the ground, allowed students to freely participate in the discussion, and in the later stage, the teacher, as the figure, helped the students to solve problems. In this mode, the teacher alternates between the role of figure and ground, which helps to arouse the enthusiasm of students to express their views freely, achieving better interaction between teachers and students.

\section{REFERENCES}

[1] Croft, W. \& D.A.Cruse. Cognitive Linguistics [M]. Beijing: Peking University Press, 2006.

[2] Harmer, J. How to Teach English [M]. Beijing: Foreign Language Teaching and Research Press, 2001

[3] Kuang Fangtao, Wen Xu, the realization of figure-ground [J]. Foreign languages, 2003,(4).

[4] Lakoff , G. \& M. Johnson. Metaphors We Live By [M]. Chicago: The University of Chicago Press, 1980

[5] Langacker, R. W. Foundations of Cognitive Grammar Vol. 1.[M]. Stanford: Stanford University Press, 1987.
[6] Liu guo-hui, the space concept of figure-ground and its metaphorical representation in language [J]. Foreign language research,2006, (2).

[7] Talmy, L. Towards a Cognitive Semantics Vol. 1.[M]. Cambridge, Mass: The MIT Press, 2000.

[8] Ungerer, F. \& H. J.Schmid. An Introduction to Cognitive Linguistics [M]. Beijing: Foreign Language Teaching and Research Press, 2001.

[9] Wen $\mathrm{xu}$ and Liu Xianqing, analysis of figure-ground theory in English inverted sentences $[\mathrm{J}]$. Foreign language teaching and research,2004,(4).

[10] Zhong Meisun, Sun Youzhong, Personnel training as the center, comprehensively promoting the reform and development of foreign language teaching, working ideas of the fifth foreign language teaching steering committee of colleges and universities [J]. Foreign language world, 2014 (1), 2-8. 